\title{
Non-reactive solute transport modelling with time-dependent dispersion through stratified porous media
}

\author{
ABHAY GULERIA*D, DEEPAK SWAMI, ABHIMANYU SHARMA and SAHIL SHARMA \\ School of Engineering, Indian Institute of Technology Mandi, Kamand 175005, India \\ e-mail: abhayguleria92@gmail.com
}

MS received 9 June 2018; revised 14 October 2018; accepted 3 December 2018; published online 16 March 2019

\begin{abstract}
We present a numerical solution of the mobile-immobile model (MIM) with time-dependent dispersion coefficient to simulate solute transport through heterogeneous porous media. Observed experimental data of non-reactive solute transport through hydraulically coupled stratified porous media have been simulated using asymptotic and linear time-dependent dispersion functions. Non-Gaussian breakthrough curves comprising long tails are simulated well with the MIM incorporating asymptotic time-dependent dispersion model. The system is under the strong influence of physical nonequilibrium, which is evident by variable mass transfer coefficient estimated at different down-gradient distances. Asymptotic time-dependent functions are capable of capturing the rising limb of the solution phase breakthrough curves with improved accuracy, whereas tailing part simulation capabilities are similar for both asymptotic and linear time-dependent dispersion functions. Further, the temporal moment analysis demonstrated increased spreading, variance for linear dispersion model as compared with asymptotic dispersion model. It is also observed that the first-order mass transfer coefficient varies inversely with travel distance from the input source. It can be concluded from the study that MIM with time-dependent dispersion function is simpler yet sensitive to account for medium's heterogeneity in a better manner even for small observation distances from the source.
\end{abstract}

Keywords. Nonreactive solute; mobile-immobile model; time-dependent dispersion; temporal moment of solute concentration; stratified porous media.

\section{Introduction}

Contaminant transport through soil has been an important research problem in the field of geo-environmental engineering. The area is still evolving with complex modelling approach and inclusion of (distance) scale- and time-dependent transport parameters. Classical advection-dispersion equation (ADE) is commonly used for describing solute transport through porous media due to its simplicity and lumped parameter estimation [1,2]. When the porous medium is heterogeneous, early arrival and long tailing of solute particles in the time-concentration plots (breakthrough curves-BTCs) have been observed, which are attributed to anomalous transport behaviour [3, 4]. It has been reported in literature that $\mathrm{ADE}$ is less adequate for simulating anomalous transport through heterogeneous porous media [3, 5-9]. Therefore, higher-order modelling approaches have been proposed that can capture anomalous transport behaviour of solute, considering physical and chemical partitioning in saturated porous media $[8,10-15]$. Physical partitioning results from the hydraulic

*For correspondence coupling of mobile (advective) and immobile (nonadvective) regions such as stratified porous media, in which the former behaves as sink/source component. Chemical partitioning results from site-specific interaction between solute and grains of porous material [10]. For the transport of nonreactive solute, mobile-immobile model (MIM) is a powerful tool. MIM divides the porous medium into flowing (mobile) and stagnant (immobile) regions. Diffusive mass transfer between mobile and immobile partitioning is mathematically accounted by mass transfer coefficient. It has been observed that the mass transfer coefficient contributes in early arrival and long tailing of BTC apart from retardation factor and dispersion coefficient [16]. In addition to this, the most challenging aspect of studying solute transport through heterogeneous porous media is ever-growing scale effect with space or time on the estimation of dispersion coefficient [17-19] since constant dispersion coefficient is a lumped parameter and unable to capture the broad spectrum of time scale BTCs through a heterogeneous porous medium [20-24]. To incorporate the scale effects, time-dependent and scale (distance)-dependent dispersion models in pre-asymptotic regime have been developed over the past decade $[8,25-28]$. Fractal concept and stochastic studies have 
established that apart from heterogeneity, travel time or distance influences the dispersion parameter until it reaches an asymptotic value [17, 18, 20, 22, 29-34]. Sharma and Srivastava [26] compared distance- and timedependent dispersion coefficients incorporated into a general transport model for physically and chemically heterogeneous porous medium. It was concluded that the distance-dependent dispersion resulted in a steeper concentration profile compared with time-dependent dispersion coefficient. Another major limitation of spacedependent dispersion coefficient is ever-growing behaviour with space [8, 20, 35]. Since the solution phase concentration rather changes with each time step, for a heterogeneous medium, time-dependent dispersion captures spreading in a better manner [9, 26, 36]. ADE incorporating time-dependent dispersion coefficient has been presented for homogeneous porous media [23, 37-40]. Basha and El-Habel [41] presented analytical solutions of ADE with arbitrary time-dependent dispersion model using variable transformation technique. There have been very few studies incorporating time-dependent dispersivity with MIM model, considering a function of mean travel distance $(\bar{x})$ [26]. In the present study, timedependent dispersion coefficient is demonstrated as a function of mean travel distance $(\bar{x})$ while in the previous studies, time-dependent dispersivity is represented by space coordinate $(x)[26,33,42,43]$. These two are not interchangeable as mean travel distance $(\bar{x})$ represents the distance of solute front at any time instance, whereas space coordinate $(x)$ is the fixed distance of the observation point from the source [27]. Hence it has been proposed that a time-dependent dispersivity should be an arbitrary function of time only [37, 41].

From literature review it is concluded that there is a need to present a novel hybrid numerical approach to solve MIM model with arbitrary time-dependent dispersion function. It is expected that MIM with time-dependent dispersion will capture the spreading in heterogeneous media, with enhanced accuracy, which increases with transport time. Moreover, it will also be interesting to see the effect of arbitrary time-dependent dispersion parameters on the plume spreading in mobile and immobile regions.

Therefore, in this study, firstly an attempt is made to extend MIM to embrace the concept of arbitrary timedependent dispersion and subsequently to solve it numerically using a hybrid implicit finite difference method. Secondly, the numerical solution developed is then used to simulate the experimental data of Swami et al [44]. Log-log plots of BTCs have been used to demonstrate the capabilities of presented solution, particularly in the rising and tailing portion. Thirdly, temporal moment analysis for linear and asymptotic time-dependent dispersion models is performed to study the plume behaviour. This study is expected to reveal the capabilities and limitations of MIM model with arbitrary time-dependent dispersion function.

\section{Governing equations}

The MIM model developed by van Genuchten and Wierenga [5, 45] is based on partitioning the heterogeneous porous media into mobile and immobile regions. Mobile region accounts for transport processes, i.e., advection and dispersion process, and stagnant or immobile region accounts for first-order diffusive mass transfer between mobile and immobile regions. Both regions include the first-order transformation reaction on specific sites in porous media. Considering linear sorption isotherm, governing equations are expressed as follows:

$$
\begin{aligned}
&\left(\theta_{m}+f \rho_{b} K_{d}\right) \frac{\partial C_{m}}{\partial t}= \theta_{m} D(t) \frac{\partial^{2} C_{m}}{\partial x^{2}}-v_{m} \theta_{m} \frac{\partial C_{m}}{\partial x} \\
&-\omega\left(C_{m}-C_{i m}\right) \\
&-\left(\theta_{m} \mu_{l m}+f \rho_{b} K_{d} \mu_{s m}\right) C_{m} \\
&\left(\theta_{i m}+(1-f) \rho_{b} K_{d}\right) \frac{\partial C_{i m}}{\partial t}=\omega\left(C_{m}-C_{i m}\right) \\
&-\left(\theta_{i m} \mu_{\text {lim }}+\right.\left.(1-f) \rho_{b} K_{d} \mu_{\text {sim }}\right) C_{i m}
\end{aligned}
$$

where $C_{m}$ and $C_{i m}$ are the solute concentrations, respectively, in the mobile and immobile regions $\left(\mathrm{M} / \mathrm{L}^{3}\right)$ at any time $\mathrm{t} ; x$ is the spatial coordinate (L) taken in the direction of the fluid flow; $D_{(t)}\left(\mathrm{L}^{2} / \mathrm{T}\right)$ represents time-dependent hydrodynamic dispersion coefficient along the flow velocity; $\theta_{m}$ and $\theta_{i m}$ are volumetric water contents of the mobile and immobile regions, respectively, and $\theta=\theta_{m}+\theta_{\text {im }} ; \theta$ is the total volumetric water content of the porous media; $v_{m}$ is the mobile pore water velocity $(\mathrm{L} / \mathrm{T}) ; v_{m} \theta_{m}$ is equal to $q$ (flow rate $(\mathrm{L} / \mathrm{T})) ; \omega$ is the first-order mass transfer coefficient $\left(\mathrm{T}^{-1}\right) ; f$ and $(1-f)$ represent the fractions of sorption sites that equilibrate instantly with the mobile and immobile regions, respectively; $\mu_{l m}$ and $\mu_{\text {lim }}$ are the first-order decay coefficients for degradation of solutes in the mobile and immobile solution phases, respectively; $\mu_{s m}$ and $\mu_{\text {sim }}$ are the first-order decay coefficients for degradation of solutes in the mobile and immobile region for adsorbed solid phases, respectively; $K_{d}$ is the distribution coefficient of the linear sorption process $\left(\mathrm{L}^{3} / \mathrm{M}\right)$ and $\rho_{b}$ is the bulk density of the porous media $\left(\mathrm{M} / \mathrm{L}^{3}\right)$.

In this study, linear and asymptotic time-dependent dispersion functions with MIM model have been used to extend the capabilities of numerical simulation. Linear time-dependent dispersion increases with time, until the end of the simulation time grid point, while asymptotic time-dependent dispersion gradually increases with time and ultimately at final time step settles with an asymptotic value. We define following abbreviations to represent our results: MIML is the MIM with linear time-dependent dispersion (LTDD); MIMA is the MIM with asymptotic time-dependent dispersion (ATDD).

Linear dispersion:

$$
D_{(t)}=D_{0} \frac{t}{K_{L}}+D_{m},
$$


Asymptotic dispersion:

$$
D_{(t)}=D_{0} \frac{t}{t+K_{A}}+D_{m}
$$

where $D_{0}$ is the maximum dispersion coefficient $\left(\mathrm{L}^{2} / \mathrm{T}\right) ; D_{m}$ is the effective diffusion coefficient $\left(\mathrm{L}^{2} / \mathrm{T}\right) ; K_{A}(\mathrm{~T})$ is the ATDD coefficient, which is equivalent to mean travel time and $K_{L}(\mathrm{~T})$ is the LTDD coefficient. It is to be noted here that in case of linear dispersion function when $K_{L}=t$, maximum dispersion coefficient is equal to total dispersion, and is 0.5 times total dispersion in case of asymptotic relation. The slope of dispersion distribution curve at any time is $D_{0} / K_{L}$ for linear function and $\frac{D_{0} K_{A}}{\left(t+K_{A}\right)^{2}}$ for asymptotic function. It shows that the gradient is constant for linear dispersion; however, in case of asymptotic function, the slope is negative. It is interesting to note that the slope of asymptotic function is equal to LTDD function at initial time and is equal to zero at infinite time. Hence, it is expected that during the initial phase of solute transport, dispersion spreading will be similar for both cases; however, later stage linear dispersion function increases with constant slope and asymptotic function reaches a constant flat line with zero slope.

\subsection{Initial and boundary conditions}

The initial condition assumes that the porous media is not contaminated and is given as follows:

$$
C_{m}(x, 0)=C_{i m}(x, 0)=0 .
$$

Following inlet and outlet boundary conditions have been used:

$$
\begin{gathered}
C_{m}(0, t)=\left\{\begin{array}{cc}
C_{0}, & 0<t \leq t_{p} \\
0, & t>t_{p}
\end{array}\right. \\
\left.\frac{\partial C_{m}(x, t)}{\partial x}\right|_{x=L}=0
\end{gathered}
$$

where $C_{0}$ is concentration $\left(\mathrm{M} / \mathrm{L}^{3}\right)$ of injected solute source at the inlet of the porous media.

\section{Development of numerical model and its validation}

In the present model, transport of non-reactive solute through saturated porous media is described using coupled partial differential equations. An implicit finite-difference formulation is used to obtain the numerical solution of MIM transport equation with time-dependent dispersion. The advection term in the mobile transport equation has been discretized using fully implicit first-order upwind scheme and dispersive term in the mobile transport equation has been discretized using second-order central difference scheme. The temporal terms of both Eqs. (1) and (2) have been discretized using a first-order forward difference scheme. Discretization of partial differential equations for non-reactive solute transport in the MIM domain is presented as follows:

$$
\begin{aligned}
R_{m}\left(\frac{C_{m_{i}}^{t+1}-C_{m_{i}}^{t}}{\Delta t}\right)= & \theta_{m} D_{(t)}\left(\frac{C_{m_{i+1}}^{t+1}-2 C_{m_{i}}^{t+1}-C_{m_{i-1}}^{t+1}}{\Delta x^{2}}\right) \\
& -v_{m} \theta_{m}\left(\frac{C_{m_{i}}^{t+1}-C_{m_{i-1}}^{t+1}}{\Delta x}\right) \\
& -\omega\left(C_{m_{i}}^{t+1}-C_{i m_{i}}^{t+1}\right)-A_{1} C_{m_{i}}^{t+1} \\
R_{i m}\left(\frac{C_{i m_{i}}^{t+1}-C_{i m_{i}}^{t}}{\Delta t}\right) & =\omega\left(C_{m_{i}}^{t+1}-C_{i m_{i}}^{t+1}\right)-A_{2} C_{i m_{i}}^{t+1}
\end{aligned}
$$

where

$$
\begin{gathered}
R_{m}=\left(\theta_{m}+f \rho_{b} K_{d}\right) \\
R_{i m}=\left(\theta_{i m}+(1-f) \rho_{b} K_{d}\right) \\
A_{1}=\left(\theta_{m} \mu_{l m}+f \rho_{b} K_{d} \mu_{s m}\right) \\
A_{2}=\left(\theta_{i m} \mu_{\text {lim }}+(1-f) \rho_{b} K_{d} \mu_{\text {sim }}\right)
\end{gathered}
$$

where $\Delta t$ represents the time step; $\Delta x$ represents the grid size; $t$ represents the known time and $(t+1)$ represents the unknown time level.

$$
\begin{aligned}
& \left(A_{7}\right) C_{m_{i}}^{t+1}-\left(A_{3} D_{(t)}\right) C_{m_{i+1}}^{t+1}-\left(A_{3} D_{(t)}+A_{4}\right) C_{m_{i-1}}^{t+1} \\
& -\left(A_{5}\right) C_{i m_{i}}^{t+1} \\
& =C_{m_{i}}^{t} \\
& \multicolumn{1}{c}{\left(A_{8}\right) C_{i m_{i}}^{t+1}-\left(A_{9}\right) C_{m_{i}}^{t+1}=C_{i m_{i}}^{t}}
\end{aligned}
$$

where coefficients are defined as follows:

$$
\begin{aligned}
& A_{3}= \frac{\theta_{m}}{(\Delta x)^{2}}\left(\frac{\Delta t}{R_{m}}\right) ; \quad A_{4}=\frac{v_{m} \theta_{m}}{\Delta x}\left(\frac{\Delta t}{R_{m}}\right) ; \\
& A_{5}=\frac{\omega \Delta t}{R_{m}} ; \quad A_{6}=\frac{A_{1} \Delta t}{R_{m}} ; \\
& A_{7}=1+\left(2 A_{3} D_{(t)}\right)+A_{4}+A_{5}+A_{6} ; \\
& A_{8}=1+\left(\frac{\omega \Delta t}{R_{\text {im }}}\right)+\left(\frac{A_{2} \Delta t}{R_{\text {im }}}\right) ; \quad A_{9}=\left(\frac{\omega \Delta t}{R_{\text {im }}}\right)
\end{aligned}
$$

$D_{(t)}$ is the time-dependent dispersion coefficient, which is updated after each time step in the numerical simulation. The discretized partial differential equations for solute transport through MIM domain have been solved using Thomas algorithm. The procedure used for simulation is shown in figure 1.

The presented numerical solution is validated with the solutions of Basha and El-Habel [41] by scaling down MIM into ADE. It is assumed that there is vanishing solute concentration in the porous media initially and a continuous 


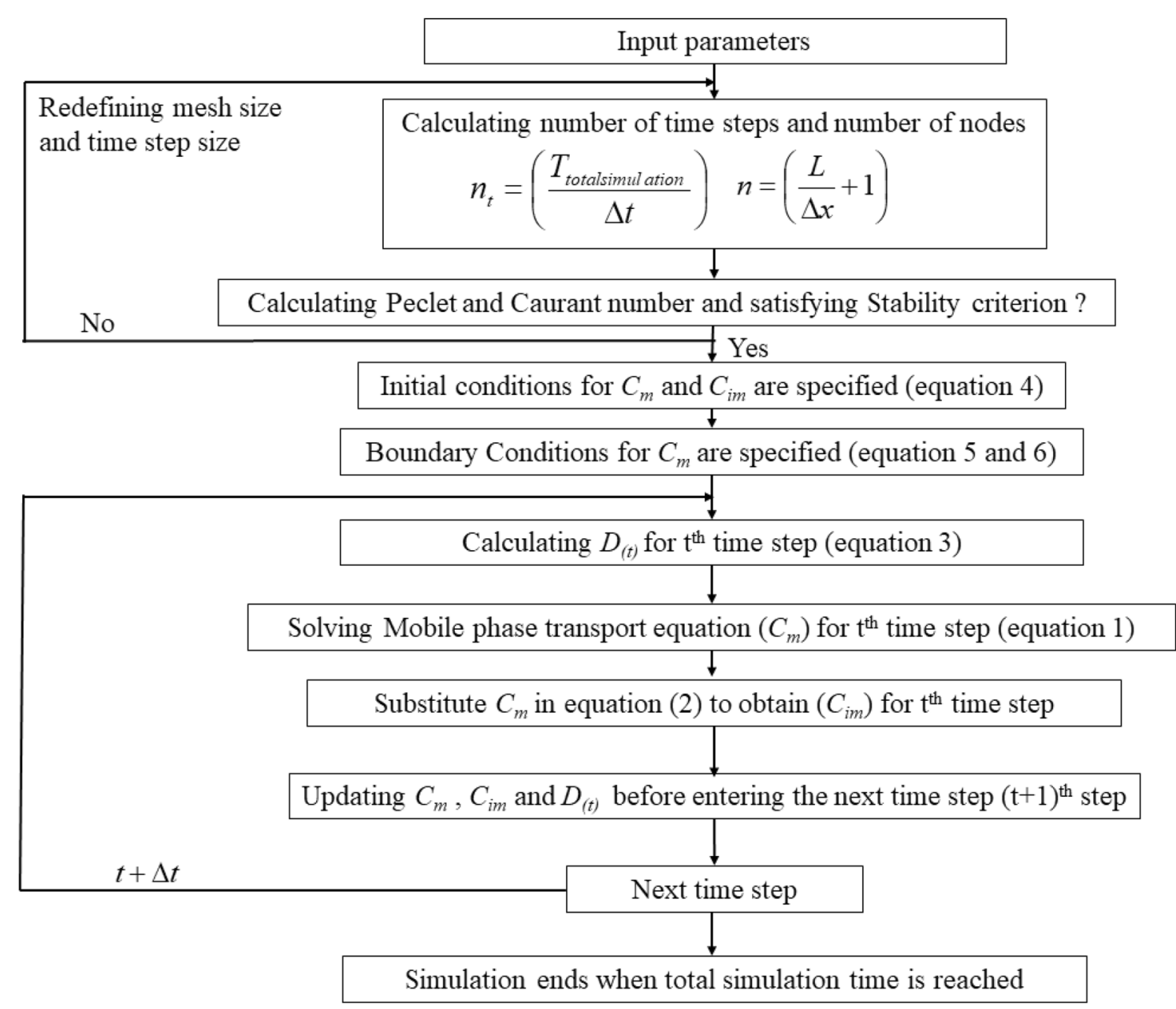

Figure 1. Flowchart of the computational algorithm to solve solute transport equations of MIM model with time-dependent dispersion.

solute source is present at the input end. For validation, input model parameters used are as follows: $L=100 \mathrm{~m}$, saturated porosity $=0.35$, pore-water velocity $=0.25 \mathrm{~m} /$ day, total time $=200$ days, retardation factor $(R)=1$, saturated porous media bulk density $\left(\rho_{b}\right)=2.11 \mathrm{~g} / \mathrm{cm}^{3}$, maximum dispersion coefficient $\left(D_{0}\right)=1 \mathrm{~m}^{2} /$ day, ATDD coefficient $\left(K_{A}\right)=50$, effective molecular diffusion coefficient $\left(D_{m}\right)=0 \mathrm{~m}^{2} /$ day and first-order decay constant $=0$ day $^{-1}$.

Figure 2 presents the variation of relative concentration for continuous injection at $T=200$ days. Relative concentration decreases gradually with distance, which is due to the dispersion processes. The numerical model shows good agreement with results obtained by Basha and El-Habel [41].

\section{Model application and evaluation}

\subsection{Simulation of experimental data of chloride transport through stratified porous media}

Mobile-immobile models with linear (MIM-LTDD) and asymptotic time-dependent dispersion (MIM-ATDD) are used to simulate observed BTCs. The experimental data of chloride transport through stratified porous media from Swami et al [44] are considered for simulation. The transport parameters such as time-dependent dispersion coefficient $\left(K_{A}\right.$ or $\left.K_{L}\right)$, maximum dispersion coefficient $\left(D_{0}\right)$ and mass-transfer coefficient $(\omega)$ have been optimized for different down-gradient distances using simulation. Experimental BTCs have been simulated at various down-gradient distances (50, 100, 150, 200, 300 and $400 \mathrm{~cm}$ ) for pore-water velocity $\left(v_{m}\right)=0.121 \mathrm{~cm} /$ $\min$.

The experimental process on the stratified porous media has been defined in Swami et al [44]. Values of physical input parameters are kept the same, i.e., mass fraction of sorbed phase that equilibrates instantly with the mobile region $(f)$ and dry bulk density $\left(\rho_{b}\right)$ of the porous media have been obtained by empirical relations given by Brusseau et al [10]:

$$
\begin{gathered}
\theta=\theta_{m}+\theta_{i m} \\
f=\left(\theta_{m} / \theta\right)
\end{gathered}
$$




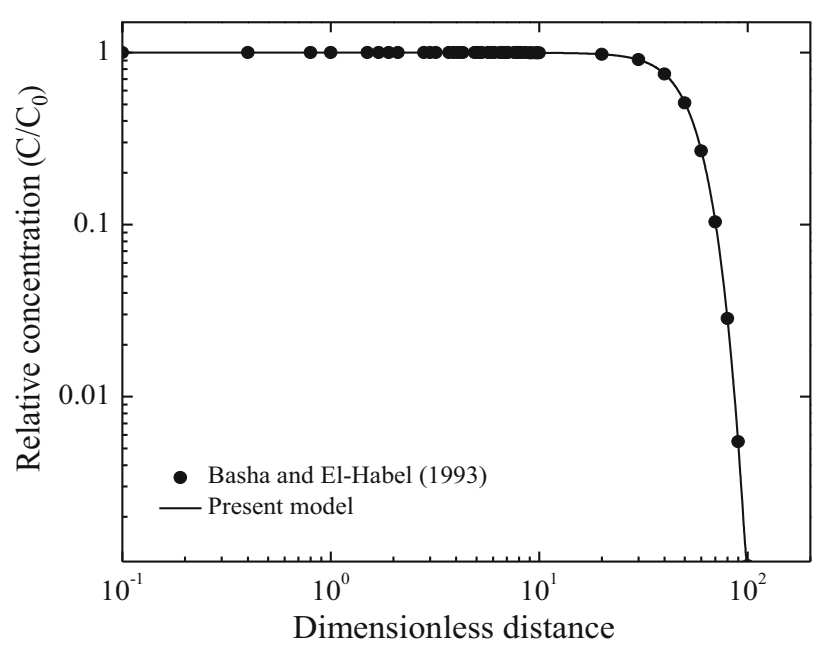

Figure 2. Concentration profile for non-reactive solute at time $T=200$ for $K_{A}=50$.

$$
\rho_{b}=f \rho_{m}+(1-f) \rho_{i m}
$$

where $\rho_{m}$ and $\rho_{i m}$ are the density of mobile and immobile regions, respectively. Using these relations, the value of $f=$ 0.44 has been used in the simulation of all experiments; $\rho_{m}$ $=1.65 \mathrm{~g} / \mathrm{cm}^{3}$ and $\rho_{\text {im }}=1.42 \mathrm{~g} / \mathrm{cm}^{3}$ are the values of density for mobile and immobile regions, respectively. Bulk density $\left(\rho_{b}\right)=1.5212 \mathrm{~g} / \mathrm{cm}^{3}$ has been calculated using Eq. (12c). The values of porosities in mobile and immobile zones obtained, respectively, are $\theta_{m}=0.33$ and $\theta_{\text {im }}=0.42$. $L=400 \mathrm{~cm}$, total simulation time $(t)=10080 \mathrm{~min}$ and a pulse duration $\left(t_{p}\right)$ of $2880 \mathrm{~min}$ at inlet have been considered.

BTCs at different down-gradient distances are simulated separately for all the dispersion models to observe the influence of travel distance on time-dependent dispersion parameters $\left(D_{0}\right.$ and $K_{L}$ or $\left.K_{A}\right)$. Estimated model parameters at all down-gradient distances for MIML and MIMA models are listed in table 1. Figures 3, 4, 5, 6, 7 and 8 show the simulation of experimental data of chloride obtained at $50,100,150,200,300$ and $400 \mathrm{~cm}$ down-gradient

Table 1. Estimated values of parameters at different down-gradient distances.

\begin{tabular}{|c|c|c|c|c|c|}
\hline \multirow[b]{2}{*}{$\begin{array}{l}\text { Distance } \\
(\mathrm{cm})\end{array}$} & \multirow[b]{2}{*}{$\begin{array}{l}\text { Mass transfer } \\
\text { coefficient } \omega \\
\quad\left(\min ^{-1}\right)\end{array}$} & \multicolumn{2}{|c|}{ MIMA } & \multicolumn{2}{|c|}{ MIML } \\
\hline & & $\begin{array}{c}D_{0} \\
\left(\mathrm{~cm}^{2} /\right. \\
\min )\end{array}$ & $\begin{array}{c}K_{A} \\
(\mathrm{~min})\end{array}$ & $\begin{array}{c}D_{0} \\
\left(\mathrm{~cm}^{2} /\right. \\
\min )\end{array}$ & $\begin{array}{c}K_{L} \\
(\mathrm{~min})\end{array}$ \\
\hline 50 & $2.8 \mathrm{E}-04$ & 1.20 & 550 & 1.32 & 5000 \\
\hline 100 & $1.7 \mathrm{E}-04$ & 1.40 & 500 & 2.00 & 5000 \\
\hline 150 & $7.2 \mathrm{E}-05$ & 1.50 & 450 & 2.77 & 4500 \\
\hline 200 & $4.75 \mathrm{E}-05$ & 3.20 & 400 & 4.97 & 3800 \\
\hline 300 & $2.95 \mathrm{E}-05$ & 3.20 & 220 & 5.04 & 4400 \\
\hline 400 & $2.12 \mathrm{E}-05$ & 4.51 & 200 & 5.89 & 4500 \\
\hline
\end{tabular}

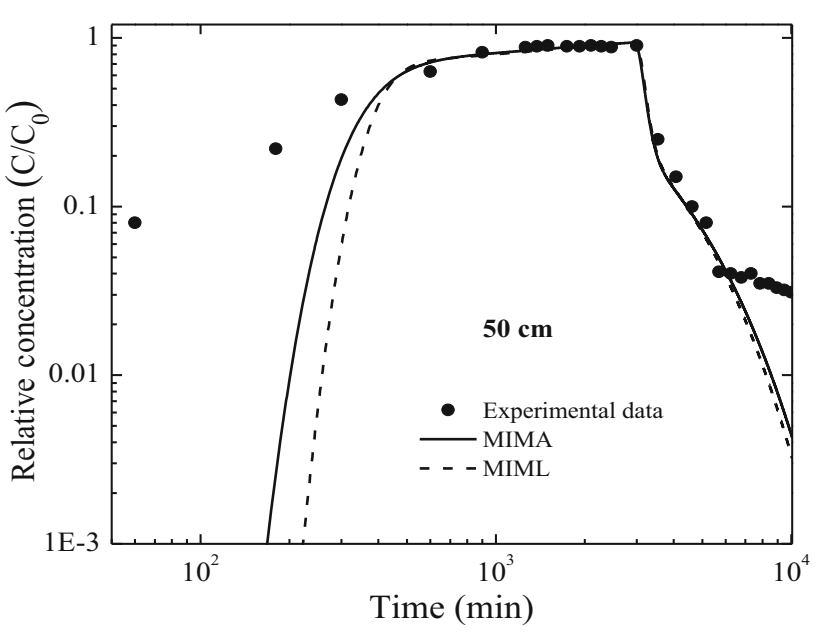

Figure 3. Simulation of experimental breakthrough curve of chloride at $50 \mathrm{~cm}$ down-gradient distance for $v_{m}=0.121 \mathrm{~cm} / \mathrm{min}$.

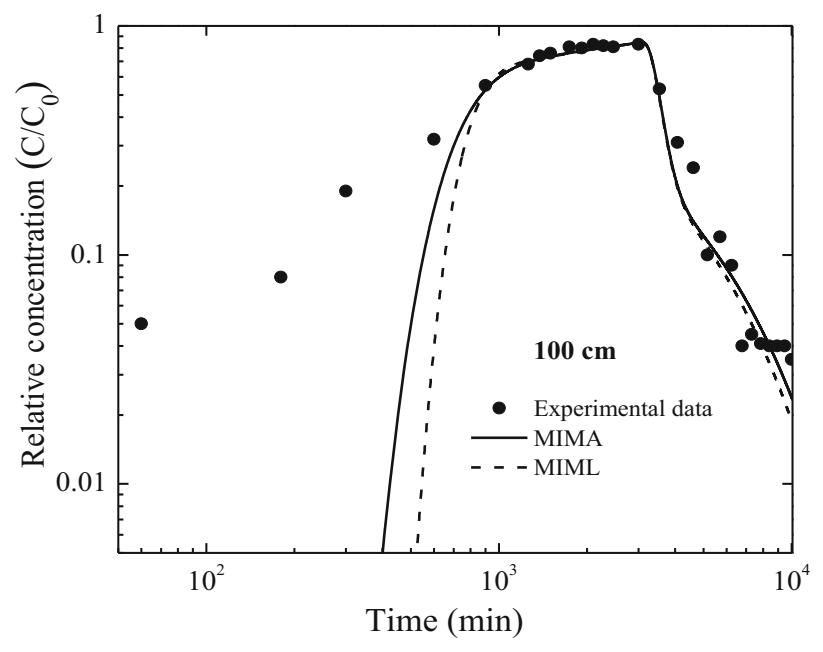

Figure 4. Simulation of experimental breakthrough curve of chloride at $100 \mathrm{~cm}$ down-gradient distance for $v_{m}=0.121 \mathrm{~cm} / \mathrm{min}$.

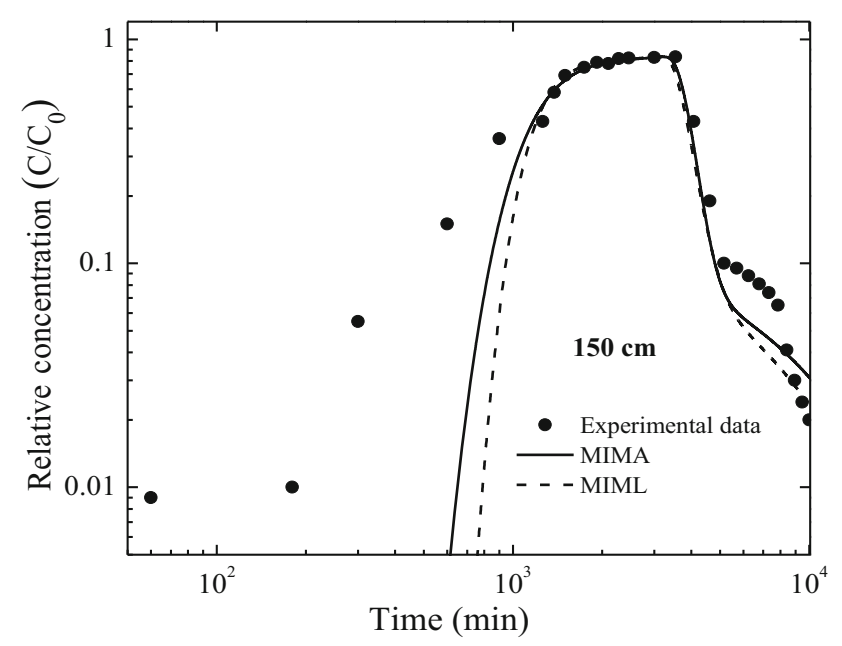

Figure 5. Simulation of experimental breakthrough curve of chloride at $150 \mathrm{~cm}$ down-gradient distance for $v_{m}=0.121 \mathrm{~cm} / \mathrm{min}$. 


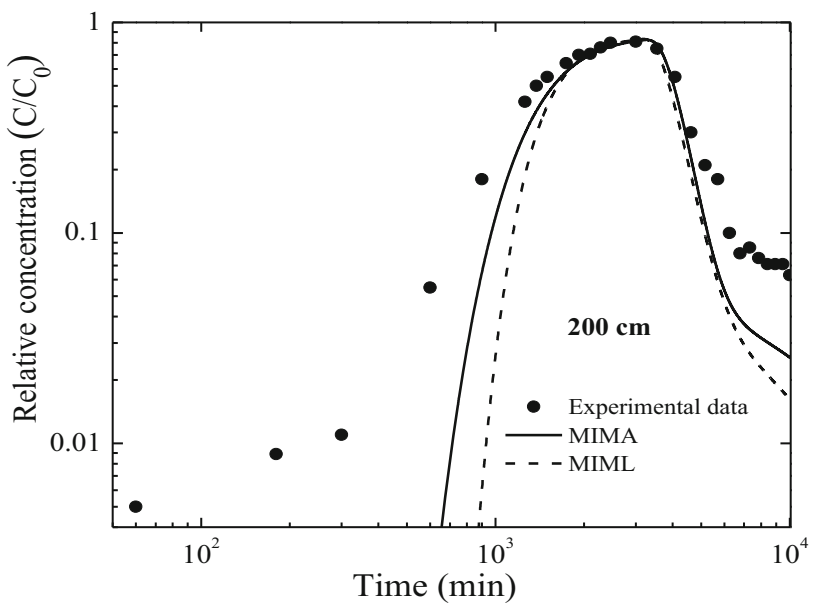

Figure 6. Simulation of experimental breakthrough curve of chloride at $200 \mathrm{~cm}$ down-gradient distance for $v_{m}=0.121 \mathrm{~cm} / \mathrm{min}$.

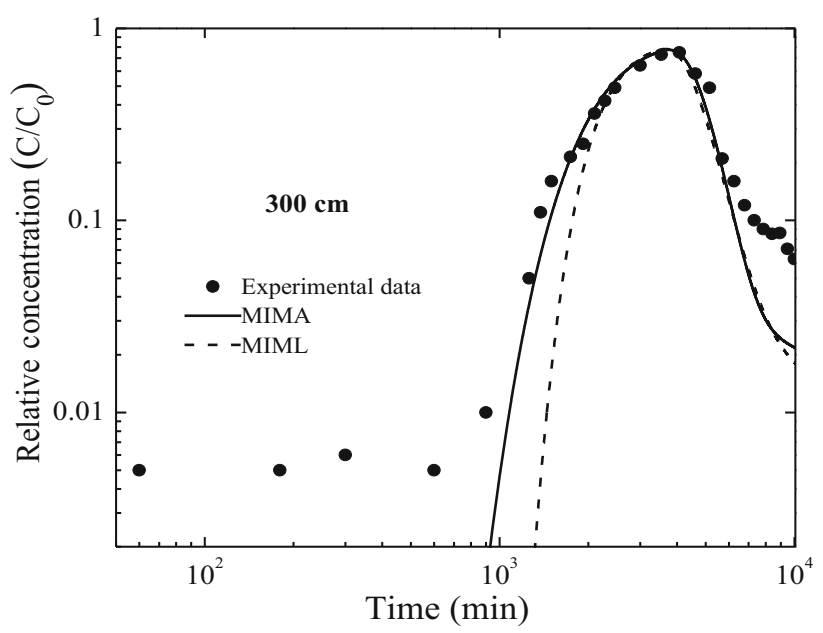

Figure 7. Simulation of experimental breakthrough curve of chloride at $300 \mathrm{~cm}$ down-gradient distance for $v_{m}=0.121 \mathrm{~cm} / \mathrm{min}$.

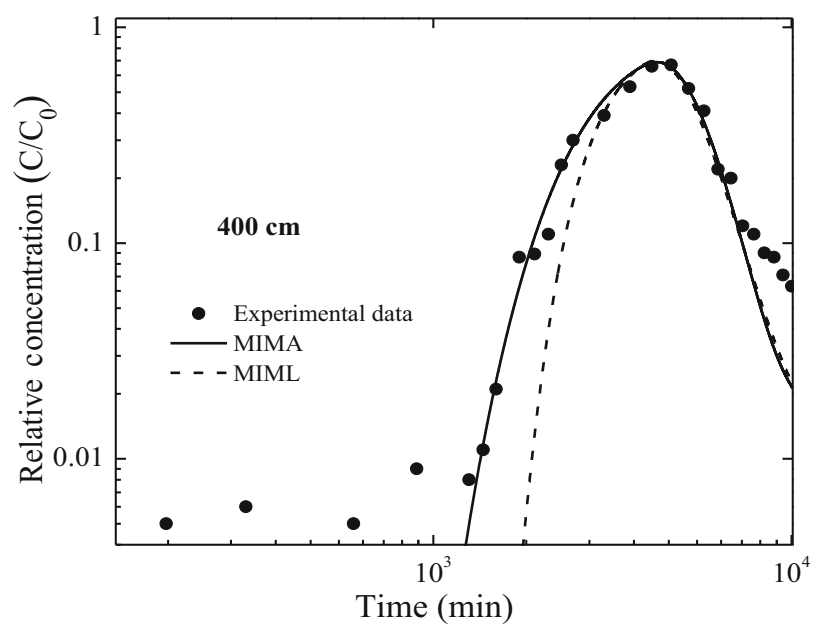

Figure 8. Simulation of experimental breakthrough curve of chloride at $400 \mathrm{~cm}$ down-gradient distance for $v_{m}=0.121 \mathrm{~cm} / \mathrm{min}$.
Table 2. The goodness of fit obtained from simulation of chloride data.

\begin{tabular}{lcllllll}
\hline & & \multicolumn{6}{c}{ Down-gradient distance $(\mathrm{cm})$} \\
\cline { 3 - 8 } Model & $\begin{array}{c}\text { Fitting } \\
\text { criterion }\end{array}$ & 400 & 300 & 200 & 150 & 100 & 50 \\
\hline MIMA & $r^{2}$ & 0.98 & 0.97 & 0.98 & 0.98 & 0.98 & 0.98 \\
& RMSE & 0.034 & 0.048 & 0.063 & 0.060 & 0.061 & 0.069 \\
& NSE & 0.97 & 0.96 & 0.95 & 0.97 & 0.96 & 0.97 \\
MIML & $r^{2}$ & 0.96 & 0.95 & 0.95 & 0.96 & 0.96 & 0.96 \\
& RMSE & 0.055 & 0.071 & 0.094 & 0.078 & 0.076 & 0.089 \\
& NSE & 0.93 & 0.91 & 0.90 & 0.94 & 0.95 & 0.95 \\
\hline
\end{tabular}

distances. Since early breakthrough and long tails contribute to the skewness of BTCs, log-log plots have been used to demonstrate the model capabilities to simulate at different down-gradient distances. Further, to compare the performance of MIMA and MIML models, root-meansquare-error (RMSE), coefficient of correlation $\left(r^{2}\right)$ and Nash-Sutcliffe efficiency (NSE) coefficient are used as criteria to reflect the goodness of simulation. Table 2 shows the goodness of fitting criterion values for all down-gradient distances.

$$
\begin{gathered}
R M S E=\sqrt{\frac{1}{n} \sum_{i=1}^{n}\left(C_{i o b s}-C_{i s i m}\right)^{2}} \\
r^{2}=\left(\frac{\sum_{i=1}^{n}\left(C_{i o b s}-\bar{C}_{i o b s}\right)\left(C_{i s i m}-\bar{C}_{i s i m}\right)}{\sqrt{\sum_{i=1}^{n}\left(C_{i o b s}-\bar{C}_{i o b s}\right)^{2}} \sqrt{\sum_{i=1}^{n}\left(C_{i s i m}-\bar{C}_{i s i m}\right)^{2}}}\right)^{2} \\
N S E=1-\left(\frac{\sum_{i=1}^{n}\left(C_{i o b s}-C_{i s i m}\right)^{2}}{\sum_{i=1}^{n}\left(C_{i o b s}-\bar{C}_{i o b s}\right)^{2}}\right)
\end{gathered}
$$

where $C_{i o b s}$ and $C_{i s i m}$ are the $i$ th observed and simulated concentration, respectively; $\overline{C_{i o b s}}$ and $\overline{C_{i s i m}}$ are the mean of observed and simulated concentration, respectively, and $n$ represents the total number of observations taken at any particular observational point.

A skewed shape of experimental and simulated results has been obtained as shown in figures 3, 4, 5, 6, 7 and 8, which is a clear demonstration that the system is under the strong influence of physical nonequilibrium. In the experimental set-up of Swami et al [24], the width of the immobile region is large and that is the reason behind the long tails with a high concentration at the concluding part of the experiments. MIM with time-dependent dispersion has demonstrated capabilities to simulate such complex scenario in pre-asymptotic regime. Figures 3, 4, 5, 6, 7 and 8 show the $\log -\log$ plot clearly accentuating the minuscule 
temporal concentration, which linear plots fail to display, particularly in breakthrough and tailing portions in such cases.

Figure 3 shows the BTC at $50 \mathrm{~cm}$ down-gradient distance; the broad peak shows the longer availability of concentration source due to proximity to the source. Hence, initial breakthrough concentration is higher and tailing portion concentration is low compared with other distances, since most of the solute particles tend to elude due to maximum exposure to fresh water source. Both MIML and MIMA demonstrated similar simulation capabilities at 50 and $100 \mathrm{~cm}$; however, MIMA model simulates initial breakthrough and tailing concentrations with slightly higher accuracy compared with MIML.

Figures 5, 6, 7 and 8 show the BTCs at $150-400 \mathrm{~cm}$ down-gradient distances; in all the curves a substantial difference between breakthrough time prediction by MIMA and MIML can be observed, where lagging of the latter increases with distance. Observation points of figures 5 and 6 placed at the centre displayed a considerable difference in prediction by MIML and MIMA in tailing portion. Also, it should be noted that the concentration in the tailing portion of the BTCs is higher towards the outlet. These effects are obviously concealed in linear-linear plots representation of BTCs many times. Hence, due to log-log representation, the limitation of MIM model with time- or scale-dependent dispersion model is truly highlighted here; it fails to capture the tailing portion of the BTCs at latter distances. A possible explanation can be that since MIM contains first-order value of mass transfer coefficient, it can be optimized to target only a specific portion of the BTC. As in many of the cases the target concentration is the peak value; hence MIM with first-order mass transfer value will fail to capture the tail portion; however, in this case, it is clear that due to inclusion of time-dependent dispersion, overall capabilities of MIM model can be enhanced to a greater extent.

It is clear from the analysis that high time-dependent dispersion coefficient $\left(K_{A}\right)$ of MIMA model is obtained for the shorter distance from input source, which is attributed to lower overall dispersion coefficient. Value of dispersion coefficient should be higher at large travel distance, which is further attributed to smaller value of time-dependent dispersion coefficient $\left(K_{A}\right)$ at large travel distance. It is evident from table 1 that the MIM with time-dependent dispersion is a powerful tool to capture observed BTCs with a highly complex experimental model with sufficiently large immobile matrix. It is also observed that due to the presence of large immobile matrix, a significant amount of concentration source is stored in an immobile matrix and it keeps on eluding till the infinite time, reaching an asymptotic state. From the BTCs and table 1 it can be concluded that the first-order diffusive mass transfer cannot be a unique value; rather it depends on the distance from the source and the time of experimentation. In the next section, temporal moment analysis is presented to provide insight of the plume behaviour within the media.

\section{Temporal moment analysis}

In the problem of contaminant transport through heterogeneous porous media, method of moments is often useful to describe the skewed BTCs at a given location. Moment analysis is even found to be a powerful tool for inverse modelling in estimating the transport parameters such as pore-water velocities and dispersion coefficients [46-49]. The difference in hydraulic conductivity causes non-ideal solute transport behaviour through heterogeneous porous media and results in a skewed BTC and higher order of first normalized temporal [50]. The zeroth moment of solute concentration represent the total mass in the system at any time instant. The first and second normalized moment of solute concentration identifies, respectively, the mean breakthrough time and the degree of spreading at any assumed location. For a condition during the experiment when a singular pulse is replaced with a clean source, the zeroth absolute temporal moment of solute concentration will start decreasing with the increase in travel distance $[51,52]$. From the moment analysis, it is identified that the total solute mass recovery is less for a larger value of masstransfer coefficient. Therefore, the performance of MIMA and MIML dispersion models has been evaluated using temporal moment analysis in this study. Also, sensitivity analysis of time-dependent dispersion coefficients (MIMA and MIML) is performed using zeroth, first and second temporal moment of solute concentration.

\subsection{Calculation of temporal moments}

Temporal moment of solute concentration provides timeaveraged response of solute behaviour in the soil [53]. The $n$ th-order absolute temporal moment of a BTC at a location $x$ is defined as

$$
M_{n}=\int_{0}^{T} t^{n} C(x, t) d t
$$

where for $n=0,1,2$, obtained moments $M_{0}, M_{1}$ and $M_{2}$, respectively represent zeroth, first and second absolute temporal moments and $T$ is the total residence time; $n$ thorder normalized temporal moment of solute concentration is given by following equations:

$$
\begin{gathered}
\mu_{n}=\frac{M_{n}}{M_{0}}=\frac{\int_{0}^{T} t^{n} C(x, t) d t}{\int_{0}^{T} C(x, t) d t} \\
T_{1}(x)=\mu_{1} \\
T_{2}(x)=\mu_{2}-\left(\mu_{1}\right)^{2}
\end{gathered}
$$


where $\mu_{1}$ and $\mu_{2}$ are the normalized first and second temporal moments, respectively. $T_{1}(x)$ and $T_{2}(x)$ are the first normalized temporal moment and second central temporal moments, respectively. The first normalized temporal moment is descriptor of mean breakthrough time, which is the average time spent by solute molecules inside the porous media. The second central temporal moment represents the variance of the BTC, which provides a measure of solute spreading.

\subsection{Comparison of MIMA and MIML using temporal moment analysis}

In this section, temporal moment of solute concentration has been compared for different time-dependent functions used with MIM model. The temporal moment analysis technique reveals the behaviour of solute plume through the porous media. Input parameters used for the temporal moment techniques are pore-water velocity $\left(v_{m}\right)$ $=0.121 \mathrm{~cm} / \mathrm{min}$, mass transfer coefficient $(\omega)=2.12 \mathrm{e}-$ $05 \mathrm{~min}^{-1}, \quad L=400 \mathrm{~cm}$, total simulation time $(t)=10080 \mathrm{~min}$, a pulse duration $\left(t_{p}\right)$ of $2880 \mathrm{~min}$, $K_{A}=200$ and $K_{L}=4500$ for MIMA and MIML model, respectively, and $D_{0}=4.51 \mathrm{~cm}^{2} / \mathrm{min}$ and $D_{0}=5.89 \mathrm{~cm}^{2} /$ min for MIMA and MIML model, respectively; figure 9a, b and $\mathrm{c}$ presents the zeroth, first and second temporal moments.

Figure 9a shows comparison of the zeroth normalized temporal moment of solute concentration for linear and ATDD functions. It is evident from figure 9a that the zeroth temporal moment of solute concentration decreases linearly with travel distance for both the MIML and MIMA models. However, at large travel distances, zeroth temporal moment of solute concentration for MIMA model attains an asymptotic value while for MIML it keeps on decreasing. Since the system is under the influence of strong physical nonequilibrium and experimental BTC having a long tail at the concluding part of the experiments, it is observed that the total mass recovery at the outlet can be better represented by MIMA compared to MIML. This is due to linear variation of dispersion with time for MIML model in comparison to MIMA model. In MIMA model, dispersion varies non-uniformly with time and reaches to an asymptotic value at higher transport time. The first temporal moment analysis in next figure is carried out to identify the average time spent by solute particles in the system for each distance.

Figure $9 \mathrm{~b}$ shows the spatial variation of first temporal moment of solute concentration and it is clear that at small travel distance, temporal moment is linear for both the models. At large travel distances, first temporal moment of solute concentration varies non-linearly for both the models, higher in the case of MIML dispersion model. The pattern of the first moment reveals the physical evergrowing nature of the MIML model with time. This shows

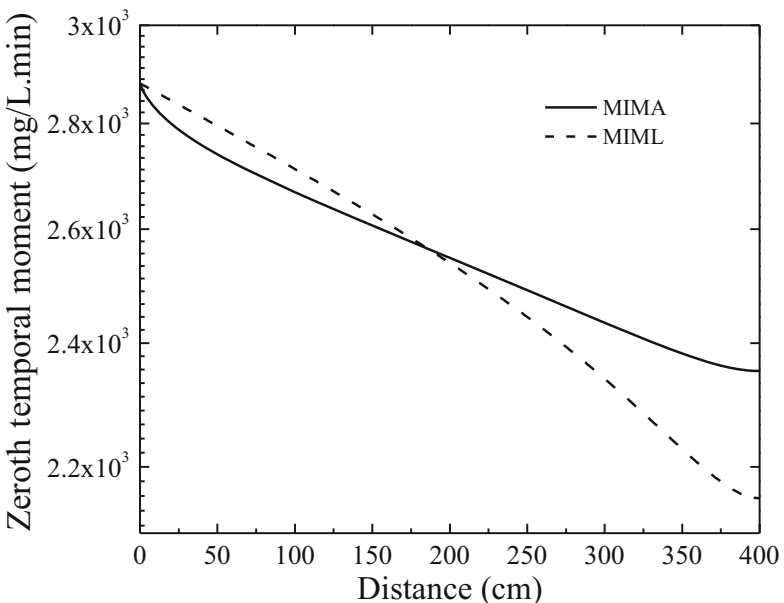

(a)

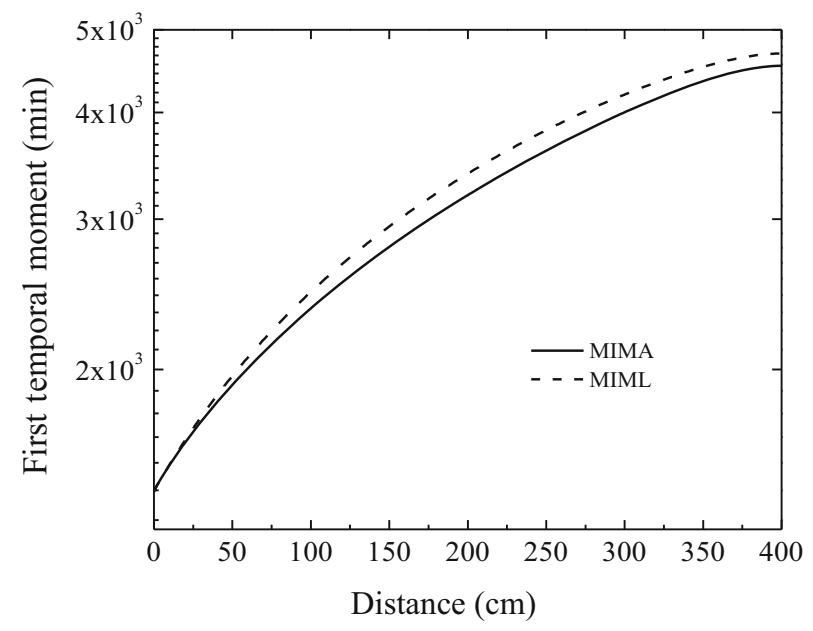

(b)

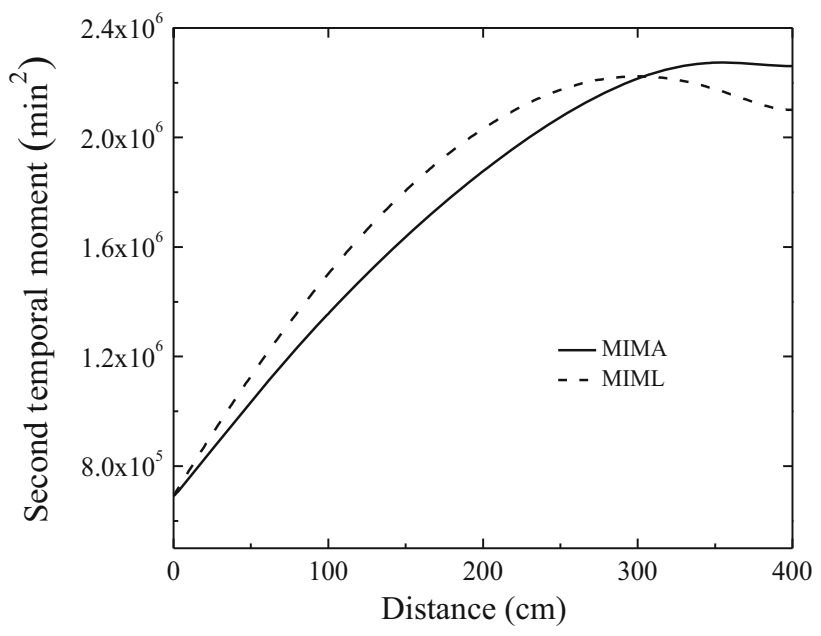

(c)

Figure 9. (a) Spatial distribution of zeroth temporal moment for MIMA and MIML. (b) Spatial distribution of first normalized temporal moment for MIMA and MIML. (c) Spatial distribution of second temporal moment for MIMA and MIML. 
that the applicability of the time-dependent dispersion is appropriate for smaller distances or experimental duration, but for the field scale, it tends to demonstrate nonrealistic representation of the plume behaviour without any upper bound. In the next figure, spatial variation of second temporal moment of solute concentration is analysed for both MIML and MIMA dispersion models.

It is evident from figure $9 \mathrm{c}$ that the second temporal moment of solute concentration for MIMA and MIML models increases non-linearly with travel distance. The variance of BTC attains an asymptotic peak value for MIMA at large travel distance; however, it decreases for MIML model. MIML tends to depart from the mean, which is due to its linear dependence on time of experimentation, which affects the plume without any upper bound. The moment analysis provides a useful insight into the plume behavior, which BTCs fall short in describing. From the moment analysis, it can be concluded that the MIML is not useful for higher experimentation time as it shows less mass recovery, higher residence time and a significant departure from the average plume moment.

\section{Conclusions}

This study presented numerical solution of MIM model with arbitrary LTDD and ATDD functions. In the case of time-dependent dispersion function, it is emphasized that the mean travel distance and space coordinate are not interchangeable. MIM model with time-dependent dispersion function showed enhanced accuracy for simulating experimental BTCs of non-reactive solute transport through stratified porous media. However, it is revealed in the loglog plot that the MIM incorporating time-dependent dispersion function fails to capture the tailing portion of BTCs. MIM embraced with time-dependent dispersion is a powerful tool, but one must prioritize the target concentration and optimize the model parameters accordingly. In this study, capturing peak concentration has been the objective function while simulating experimental BTCs. MIM model with ATDD demonstrated enhanced accuracy compared with linear dispersion (LTDD) model. Temporal moment analysis revealed the limitation of MIML at higher travel distances and endorsed the use of MIMA due to its realistic representation of spreading, skewness and mean travel time of solute plume. In future, such skewed BTCs can be analysed for rising and falling limb separately to get an insight about the first-order mass transfer coefficient in MIM incorporating time-dependent dispersion function. Additionally, in future the transport of reactive solutes through heterogeneous porous media can also be included for a realistic scenario.

\section{List of symbols}

$C_{0} \quad$ injected concentration of solute source $\left(\mathrm{M} / \mathrm{L}^{3}\right)$
$C_{m} \quad$ solute concentration in the mobile region at any time $t\left(\mathrm{M} / \mathrm{L}^{3}\right)$

$C_{i m} \quad$ solute concentration in the immobile region at any time $t\left(\mathrm{M} / \mathrm{L}^{3}\right)$

$D_{(t)} \quad$ time-dependent hydrodynamic dispersion coefficient along the flow velocity $\left(\mathrm{L}^{2} / \mathrm{T}\right)$

$D_{0} \quad$ maximum dispersion coefficient $\left(\mathrm{L}^{2} / \mathrm{T}\right)$

$D_{m} \quad$ effective diffusion coefficient $\left(\mathrm{L}^{2} / \mathrm{T}\right)$

$K_{A} \quad$ asymptotic time-dependent dispersion coefficient (T)

$K_{L} \quad$ linear time-dependent dispersion coefficient (T)

$M_{0} \quad$ zeroth absolute temporal moment of solute concentration

$M_{1} \quad$ first absolute temporal moment of solute concentration

$M_{2} \quad$ second absolute temporal moment of solute concentration

$T_{1}(x) \quad$ first normalized temporal moment of solute concentration

$T_{2}(x) \quad$ second central temporal moment of solute concentration

$\theta_{m} \quad$ volumetric water content of the mobile region

$\theta_{i m} \quad$ volumetric water content of the immobile region

$\theta$ total volumetric water content of the porous media

$v_{m} \quad$ mobile pore water velocity $(\mathrm{L} / \mathrm{T})$

$q \quad$ flow rate $(\mathrm{L} / \mathrm{T})$

$\omega \quad$ first-order mass transfer coefficient $\left(\mathrm{T}^{-1}\right)$

$f \quad$ fraction of adsorption sites that equilibrate instantly with the mobile regions

$\mu_{l m} \quad$ first-order decay coefficient for degradation of solute in the mobile solution phase $\left(\mathrm{T}^{-1}\right)$

$\mu_{\text {lim }} \quad$ first-order decay coefficient for degradation of solute in the immobile solution phase $\left(\mathrm{T}^{-1}\right)$

$\mu_{s m} \quad$ first-order decay coefficient for degradation of solute in the mobile region adsorbed solid phase $\left(\mathrm{T}^{-1}\right)$

$\mu_{\text {sim }} \quad$ first-order decay coefficient for degradation osolute in the immobile region adsorbed solid phase $\left(\mathrm{T}^{-1}\right)$

$\mu_{1} \quad$ first normalized temporal moment of solute concentration

$\mu_{2} \quad$ second normalized temporal moment of solute concentration

$K_{d} \quad$ distribution coefficient of the linear sorption $\operatorname{proc}\left(\mathrm{L}^{3} / \mathrm{M}\right)$

$\rho_{b} \quad$ bulk density of the porous medium $\left(\mathrm{M} / \mathrm{L}^{3}\right)$

$x \quad$ spatial coordinate taken in the direction of the fluid flow (L)

ADE advection-dispersion equation

ATDD asymptotic time-dependent dispersion function

LTDD linear time-dependent dispersion function

MIM mobile-immobile model

MIMA mobile-immobile model with asymptotic timedependent dispersion function 
MIML mobile-immobile model with linear timedependent dispersion function

\section{References}

[1] Bear J 1972 Dynamics of fluids in porous media. New York: Elsevier

[2] Bear J 1979 Hydraulics of groundwater. New York: McGraw-Hill International Book Co

[3] Levy M and Berkowitz B 2003 Measurement and analysis of non-Fickian dispersion in heterogeneous porous media. $J$. Contam. Hydrol. 64: 203-226. https://doi.org/10.1016/ s0169-7722(02)00204-8

[4] Cortis A and Berkowitz B 2004 Anomalous transport in "classical" soil and sand columns. Soil Sci. Soc. Am. J. 68: 1539-1548. https://doi.org/10.2136/sssaj2004.1539

[5] van Genuchten M T and Wierenga P J 1976 Mass transfer studies in sorbing porous media I. Analytical solutions. Soil Sci. Soc. Am. J. 40: 473-480. https://doi.org/10.2136/ sssaj1976.03615995004000040011x

[6] Li L, Barry D A, Cuiligan-Hensley P J and Bajracharya K 1994 Mass transfer in soils with local stratification of hydraulic conductivity. Water Resour. Res. 30: 2891-2900. https://doi.org/10.1029/94wr01218

[7] Gao G, Feng S, Zhan H, Huang G and Mao X 2009 Evaluation of anomalous solute transport in a large heterogeneous soil column with mobile-immobile model. J. Hydrol. Eng. 14: 966-974. https://doi.org/10.1061/(asce)he.1943-5584. 0000071

[8] Gao G, Zhan H, Feng S, Fu B, Ma Y and Huang G 2010 A new mobile-immobile model for reactive solute transport with scale-dependent dispersion. Water Resour. Res. 46: 1-16. https://doi.org/10.1029/2009wr008707

[9] Sharma P K, Ojha C S P and Joshi N 2014 Finite volume model for reactive transport in fractured porous media with distance- and time-dependent dispersion. Hydrol. Sci. J. 59: 1582-1592. https://doi.org/10.1080/02626667.2014.932910

[10] Brusseau M L, Jessup R E and Rao P S C 1989 Modeling the transport of solutes influenced by multiprocess nonequilibrium. Water Resour. Res. 25: 1971-1988. https://doi.org/10. 1029/wr025i009p01971

[11] Huang K, Toride N and Van Genuchten M T 1995 Experimental investigation of solute transport in large, homogeneous and heterogeneous, saturated soil columns. Transp. Porous Media 18: 283-302

[12] Kartha S A and Srivastava R 2008 Effect of slow and fast moving liquid zones on solute transport in porous media. Transp. Porous Media 75: 227-247

[13] Swami D, Sharma P K and Ojha C S P 2013 Experimental investigation of solute transport in stratified porous media. ISH J. Hydraul. Eng. 19: 145-153. https://doi.org/10.1080/ 09715010.2013.793930

[14] Joshi N, Ojha C S P, Sharma P K and Madramootoo C A 2015 Application of nonequilibrium fracture matrix model in simulating reactive contaminant transport through fractured porous media. Water Resour. Res. 51: 390-408. https://doi. org/10.1002/2014wr016500
[15] Mehmani Y and Balhoff M T 2015 Mesoscale and hybrid models of fluid flow and solute transport. Rev. Mineral. Geochem. 80: 433-459

[16] Nielsen D R, Th. Van Genuchten M and Biggar J W 1986 Water flow and solute transport processes in the unsaturated zone. Water Resour. Res. 22: 89S-108S, https://doi.org/ 10.1029/wr022i09sp0089s

[17] Gelhar L W, Welty C and Rehfeldt K R 1992 A critical review of data on field-scale dispersion in aquifers. Water Resour. Res. 28: 1955-1974. https://doi.org/10.1029/ $92 \mathrm{wr} 00607$

[18] Dagan G 1988 Time-dependent macrodispersion for solute transport in anisotropic heterogeneous aquifers. Water Resour. Res. 24: 1491-1500

[19] Zhou L and Selim H M 2003 Scale-dependent dispersion in soils: an overview. Adv. Agron. 80: 223-263

[20] Pickens J F and Grisak G E 1981 Scale-dependent dispersion in a stratified granular aquifer. Water Resour. Res. 17: $1191-1211$

[21] Zhou L and Selim H M 2003 Application of the fractional advection-dispersion equation in porous media. Soil Sci. Soc. Am. J. 67: 1079-84. https://doi.org/10.2136/sssaj2003.1079

[22] Schulze-Makuch D 2005 Longitudinal dispersivity data and implications for scaling behavior. Ground Water 43: 443-456. https://doi.org/10.1111/j.1745-6584.2005.0051.x

[23] Kumar G S, Sekhar M and Misra D 2008 Time-dependent dispersivity of linearly sorbing solutes in a single fracture with matrix diffusion. J. Hydrol. Eng. 13: 250-257, https:// doi.org/10.1061/(asce)1084-0699(2008)13:4(250)

[24] Swami D, Sharma P K and Ojha C S P 2014 Simulation of experimental breakthrough curves using multiprocess nonequilibrium model for reactive solute transport in stratified porous media. Sadhana Acad. Proc. Eng. Sci. 39: 1425-1446. https://doi.org/10.1007/s12046-014-0287-9

[25] Jaiswal D K, Kumar A, Kumar N and Yadav R R 2009 Analytical solutions for temporally and spatially dependent solute dispersion of pulse type input concentration in onedimensional semi-infinite media. J. Hydro-Environ. Res. 2: 254-263. http://dx.doi.org/10.1016/j.jher.2009.01.003

[26] Sharma P K and Srivastava R 2012 Concentration profiles and spatial moments for reactive transport through porous media. J. Hazard. Toxic Radioact. Waste 16: 125-133. https://doi.org/10.1061/(asce)hz.2153-5515.0000112

[27] Selim H 2014 Transport \& fate of chemicals in soils: principles \& applications. CRC Press, Boca Raton

[28] Guérin T and Dean D S 2017 Universal time-dependent dispersion properties for diffusion in a one-dimensional critically tilted potential. Phys. Rev. E 95: 12109

[29] Gelhar L W, Gutjahr A L and Naff R L 1979 Stochastic analysis of macrodispersion in a stratified aquifer. Water Resour. Res. 15: 1387-1397

[30] Pickens F and Grisak E 1981 Modelling of scale-dependent dispersion in hydrogeologic systems. Water Resour. Res. 17: 1701-1711

[31] Sudicky E A 1986 A natural gradient experiment on solute transport in a sand aquifer: spatial variability of hydraulic conductivity and its role in the dispersion process. Water Resour. Res. 22: 2069-2082

[32] Arya A, Hewett T A, Larson R G and Lake L W 1988 Dispersion and reservoir heterogeneity. SPE Reserv. Eng. 3: 139-148. https://doi.org/10.2118/14364-pa 
[33] Wheatcraft S W and Tyler S W 1988 An explanation of scale-dependent dispersivity in heterogeneous aquifers using concepts of fractal geometry. Water Resour. Res. 24: 566-578. https://doi.org/10.1029/wr024i004p00566

[34] Logan J D 1996 Solute transport in porous media with scaledependent dispersion and periodic boundary conditions. $J$. Hydrol. 184: 261-276. https://doi.org/10.1016/0022-1694 (95)02976-1

[35] Swami D, Sharma A, Sharma P K and Shukla D P 2016 Predicting suitability of different scale-dependent dispersivities for reactive solute transport through stratified porous media. J. Rock Mech. Geotech. Eng. 8: 921-927. https://doi. org/10.1016/j.jrmge.2016.07.005

[36] Natarajan N 2016 Effect of distance-dependent and time-dependent dispersion on non-linearly sorbed multispecies contaminants in porous media. ISH J. Hydraul. Eng. 22: 16-29

[37] Barry D A and Sposito G 1989 Analytical solution of a convection-dispersion model with time-dependent transport coefficients. Water Resour. Res. 25: 2407-2416

[38] Zou S, Xia J and Koussis A D 1996 Analytical solutions to non-Fickian subsurface dispersion in uniform groundwater flow. J. Hydrol. 179: 237-258. https://doi.org/10.1016/00221694(95)02830-7

[39] Sander G C and Braddock R D 2005 Analytical solutions to the transient, unsaturated transport of water and contaminants through horizontal porous media. Adv. Water Resour. 28: 1102-1111. https://doi.org/10.1016/j.advwatres.2004.10. 010

[40] Kumar G S, Sekhar M and Misra D 2006 Time dependent dispersivity behavior of non-reactive solutes in a system of parallel fractures. Hydrol. Earth Syst. Sci. Discuss. 3: 895-923. https://doi.org/10.5194/hessd-3-895-2006

[41] Basha H A and El-Habel F S 1993 Analytical solution of the onedimensional time-dependent transport equation. Water Resour. Res. 29: 3209-3214, https://doi.org/10.1029/93wr01038

[42] Yates S R 1990 An analytical solution for one-dimensional transport in heterogeneous porous media. Water Resour. Res. 26: $2331-2338$

[43] Yates S R 1992 An analytical solution for one-dimensional transport in porous media with an exponential dispersion function. Water Resour. Res. 28: 2149-2154
[44] Swami D, Sharma P K and Ojha C S P 2016 Behavioral study of the mass transfer coefficient of nonreactive solute with velocity, distance, and dispersion. J. Environ. Eng. 143: 1-10. https://doi.org/10.1061/(asce)ee.1943-7870.0001164

[45] van Genuchten M T and Wierenga P J 1977 Mass transfer studies in sorbing porous media: II. Experimental evaluation with tritium. Soil Sci. Soc. Am. J. 41: 272-278

[46] Yu C, Warrick A W and Conklin M H 1999 A moment method for analyzing breakthrough curves of step inputs. Water Resour. Res. 35: 3567-3572. https://doi.org/10.1029/ 1999wr900225

[47] Pang L, Goltz M and Close M 2003 Application of the method of temporal moments to interpret solute transport with sorption and degradation. J. Contam. Hydrol. 60: 123-134. https://doi.org/10.1016/s0169-7722(02)00061-x

[48] Bardsley W E 2003 Temporal moments of a tracer pulse in a perfectly parallel flow system. Adv. Water Resour. 26: 599-607. https://doi.org/10.1016/s0309-1708(03)00047-2

[49] Renu V and Kumar G S 2016 Temporal moment analysis of multi-species radionuclide transport in a coupled fractureskin-matrix system with a variable fracture aperture. Environ. Model. Assess. 21(4): 547-562. https://doi.org/10.1007/ s10666-016-9515-5

[50] Srivastava R and Brusseau M L 1996 Nonideal transport of reactive solutes in heterogeneous porous media: 1 . Numerical model development and moments analysis. J. Contam. Hydrol. 24: 117-143. https://doi.org/10.1016/s0169-7722 (96)00039-3

[51] Srivastava R, Sharma P K and Brusseau M L 2004 Reactive solute transport in macroscopically homogeneous porous media: analytical solutions for the temporal moments. $J$. Contam. Hydrol. 69: 27-43. https://doi.org/10.1016/s01697722(03)00155-4

[52] Sharma P K, Sekhar M, Srivastava R and Ojha C S P 2012 Temporal moments for reactive transport through fractured impermeable/permeable formations. J. Hydrol. Eng. 17: 1302-1314. https://doi.org/10.1061/(asce)he.1943-5584. 0000586

[53] Govindaraju R S and Das B S 2007 Moment analysis for subsurface hydrologic applications. Springer Netherlands, Dordrecht 